\title{
Is there a rationale for aggressive breast cancer liver metastases resections in Polish female patients? Analysis of overall survival following hepatic resection at a single centre in Poland
}

\author{
Eliza Kobryń1, Konrad Kobryń', Tadeusz Wróblewski', Krzysztof Kobryń1, Radosław Pietrzak',
} Paweł Rykowski', Bogna Ziarkiewicz-Wróblewska², Krzysztof Lamparskii ${ }^{3}$, Krzysztof Zieniewicz ${ }^{1}$, Waldemar Patkowski', Marek Krawczyk'1, Rafał Paluszkiewicz'

${ }^{1}$ Department of General, Transplant and Liver Surgery, Medical University, Warsaw, Poland

${ }^{2}$ Department of Pathology, Medical University, Warsaw, Poland

${ }^{3}$ Department of Radiology, Medical University, Warsaw, Poland

Kobryń E, Kobryń K, Wróblewski T, Kobryń K, Pietrzak R, Rykowski P, Ziarkiewicz-Wróblewska B, Lamparski K, Zieniewicz K, Patkowski W, Krawczyk M, Paluszkiewicz R. Is there a rationale for aggressive breast cancer liver metastases resections in Polish female patients? Analysis of overall survival following hepatic resection at a single centre in Poland. Ann Agric Environ Med. 2016; 23(4): 683-687. doi: 10.5604/12321966.1226866

\begin{abstract}
Introduction. Breast cancer (BC) makes up nearly $26 \%$ of malignant tumours worldwide and is the leading cause of cancerrelated deaths in European women. With approximately 18,000 new cases of BC diagnosed in Polish women annually, breast cancer liver metastasis (BCLM) is respectively an increasing issue. Recent data found in literature indicates improved survival following liver resection with systemic therapy.

Objective. The aim of study was to evaluate surgical treatment in patients with isolated BCLM.

Materials and method. During 2009-2013, a retrospective study was undertaken and 30 cases analysed. From nearly 2,000 liver resections performed, 11 female patients at the mean age of 59.18 years with BCLM were qualified for surgery.

Results. The median time between primary and secondary treatment was 3.5 years (1-7). One patient (9.1\%) presented an extrahepatic lesion - bone metastasis. The left lobe, right lobe and both lobes of the liver were affected, respectively, in $3(27.3 \%), 4(36.4 \%)$ and $4(36.4 \%)$ patients. 5 patients (45.5\%) presented single hepatic lesion, in contrast to the maximum number of lesions which equalled 6 in the right lobe. Average hospitalisation period was 13.27 days and discharge on the 11.3 postoperative day. One-year survival was $72.7 \%$ (8 patients); therefore, three-year survival was $36.4 \%$ (4 patients).

Conclusions. Oncological centres should assess BCLM patients more openly and qualify them for hepatic resection along with adjuvant systemic treatment in order to improve overall survival. This, however, needs to be studied in a multicentre randomized trial.
\end{abstract}

\section{Key words}

liver metastases, breast cancer, liver surgery

\section{INTRODUCTION}

Despite widespread screening programmes, breast cancer demographically remains an important oncological issue in the European Union. According to National Registry of Cancer, in Poland, breast cancer accounts for up to $21.9 \%$ of all malignant neoplasms, and is the second leading cause of tumour-related deaths in Polish women [1]. Although the mortality of breast cancer is gradually decreasing [2], it still accounts for up to $13.9 \%$ of deaths in oncological patients. The number of Polish women diagnosed with breast cancer is steadily increasing, reaching almost 18,000 new cases per year (Fig. 1). Breast cancer most frequently affects women in their post-menopause period of life. In $80 \%$ of cases, breast cancer occurs in females over 50 years of age and in approximately $50 \%$ of cases in the $6^{\text {th }}$ and $7^{\text {th }}$ decades of life [1]. The strongest risk factor for breast malignancies is the increasing age of the population, while the second is gender - the incidence in women is dominantly higher, as in men

Address for correspondence: Konrad Kobryń, MD., Dept. of General, Transplant and Liver Surgery, Medical University, Banacha 1a, 02-097 Warsaw, Poland

E-mail: konradkobryn@gmail.com;

Received: 06 December 2016; accepted: 15 December 2016

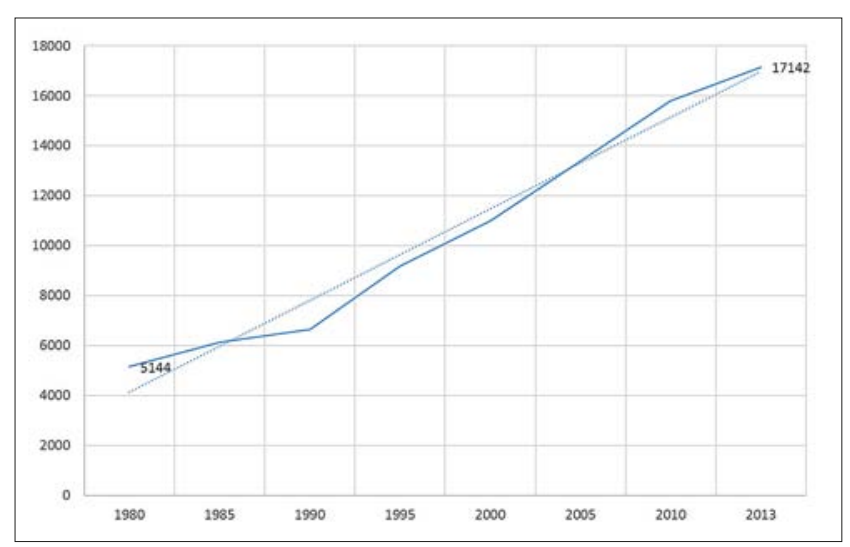

Figure 1. Breast cancer diagnosed in Polish women during the last 30 years

as it accounts for only up to $1 \%$ of all malignant tumours [1].

Other factors reported to influence the incidence of breast cancer and its morbidity are: gene mutations of BRCA1, BRCA2, familial occurrence of breast cancer, hormonal disorders and environmental reasons explained by increasing obesity due to excessive consumption of saturated fatty acids $[1,2]$. 
The treatment and prognosis of breast cancer are determined by many factors, but in the majority of cases the staging of the disease is an objective indicator for treatment options [3]. In approximately $20 \%$ of patients during their life, distant metastasis will be presented $[4,5,6]$. According to the autopsy studies carried out by Lee [7], the liver is the third most common site of breast cancer metastasis, present in $50-71 \%$ of all metastatic patients, following metastasis to the lungs (55-77\%) and bones (49-74\%).

The presence of metastasis predicts poor overall outcome in breast cancer-bearing patients [8]. The breast is the most common primary tumour location in patients with noncolorectal, non-neuroendocrine liver metastasis [9]. On the other hand, breast cancer in the stadium of a disseminated disease involves the liver in $50 \%$ of cases [6] - but only $5-25 \%$ of patients with stage IV cancer present isolated breast cancer liver metastasis (BCLM), which potentially could be radically treated by hepatic resection [10]. In other cases, the systematic and targeted therapy is the only resort for palliative treatment, which is not as effective as surgery $[11,12]$. While the response to systemic therapy in primary tumours is up to $90 \%$, in metastatic lesions it is only $50 \%$ [13]. This situation is probably caused by higher chemotherapeutic drug-resistance acquired during the course of treatment $[14,15]$. In the light of these facts, surgery may be the most beneficial treatment in patients with isolated BCLM [16].

\section{OBJECTIVE}

The study aimed to evaluate the efficacy of operational treatment, such as hepatic resection with radiofrequency ablation (RFA) or RFA alone and long-term outcome, in patients with isolated breast cancer liver metastasis, especially in the context of potential factors associated with overall survival (OS).

\section{MATERIALS AND METHOD}

Study design and patients' characteristics. A retrospective single centre study was carried out in the General, Transplant and Liver Surgery Department at the Medical University of Warsaw. Data regarding baseline characteristic of patients hospitalized in the department between 2009-2013 was retrospectively collected from medical records. The inclusion criteria were: patients with recognized and postoperatively confirmed metachronous breast cancer liver metastasis, who had previously undergone total mastectomy (Halsted or Patey's mastectomy), or breast conserving treatment (BCT). The exclusion criteria were: insufficient or incomplete medical documentation, disseminated oncological disease, disqualification from hepatic surgery due to other reasons, histopathological findings of non-BCLM, and none compliance regarding patient follow-up.

The following parameters were analysed in the search for potential factors for overall survival rate (OSR) and disease-free survival rate (DSR): gender, age, type of previous mastectomy, additional therapy, period between mastectomy and liver resection, presence of metastasis in other organs, number of lesions in the liver and number of affected segments, vascular lesions, type of liver resection, postoperative complications, average hospitalization period and time of discharge after surgery, postoperative mortality and morbidity, postoperative additional therapy and longterm outcome.

Hepatic resection. Each patient qualified for hepatic resection underwent all necessary examinations: blood tests, serum levels of creatinine, bilirubin and aminotransferases, chest X-ray, and consulted by gynaecologists, to exclude nonBCLM distant metastasis or other new neoplasms.

The radiological imaging was performed to assess the tumour's expansion in the liver. As standard preoperative work-up, an abdominal ultrasound examination and 3-phase computed tomography were performed. The results were consulted with by radiologists, surgeons, anaesthetists and oncologists. Patients were then qualified for the types of liver resection: major liver resection (three or more segments), segmentectomy, metastasectomy, hepatic resection with intraoperative RFA.

Postoperative outcome and follow-up evaluation. The postoperative mortality was defined as death within 60 days from surgery; thus, the postoperative morbidity was defined as the simultaneous adverse effects of surgery. The overall survival rate was considered as time from the surgery to the death of the patient, or date of last available follow up; the disease-free survival rate was defined as time from the date of surgery to the date of recurrence.

Follow-up was carried out by surgical staff in the outpatient clinic and a study-driven questionnaire completed during the interview.

\section{RESULTS}

From among 30 patients suspected of BCLM hospitalized during the analysed period, 11 fulfilled the inclusion criteria. They were all females.

The mean age of the study population at the time of breast cancer diagnosis was 54 years $( \pm 12.37)$. 9 patients $(81.8 \%)$ underwent total mastectomy and 2 patients (18.2\%) breast conserving treatment as the primary treatment of breast cancer. From these patients, one had bilateral breast cancer treated also by radical breast resection (Tab. 1).

The mean age at the moment of BCLM diagnosis was 59 years $( \pm 11.34)$ and the median time between primary treatment and occurrence of liver metastasis - 3.5 years (1-7). One patient (9.1\%) presented an extrahepatic lesion bone metastasis. The left lobe, right lobe and both lobes of liver were affected, respectively, in 3 (27.3\%), 4 (36.4\%) and 4 (36.4\%) patients. 5 patients $(45.5 \%)$ presented single hepatic lesion, in contrast to the maximum number of lesions which equalled 6 in the right lobe.

Hepatic resection. All performed laparotomies were primary hepatic resections. Among 11 patients qualified for liver resection, 10 resections were performed and one patient underwent RFA. In most cases (6 patients - 54.5\%), the surgery was limited to segmentectomy or metastasectomy. $4(36.4 \%)$ of 10 resections were performed with associating RFA. Histopathology examination showed BCLM with negative oncological margins in all resected specimens. The characteristic of liver resection is presented in Table 2. 
Table 1. Patient and tumour characteristics $(n=11)$

\begin{tabular}{|c|c|c|c|}
\hline \multicolumn{2}{|l|}{ Primary breast tumour } & $\mathrm{n}$ & \multirow{2}{*}{$\begin{array}{c}\% \\
(52.5)\end{array}$} \\
\hline & Mean age (M) & $54 \pm 12.37$ & \\
\hline \multicolumn{4}{|l|}{ Surgical treatment } \\
\hline & Breast conserving treatment & 2 & 18.2 \\
\hline & Total (Patey's) mastectomy & 9 & 81.8 \\
\hline \multicolumn{4}{|l|}{ Systemic treatment } \\
\hline & Adjuvant chemotherapy & 6 & 66.7 \\
\hline & Adjuvant radiotherapy & 4 & 44.4 \\
\hline & Adjuvant hormone therapy & 4 & 44.4 \\
\hline & Database unavailable & 2 & 18.2 \\
\hline \multicolumn{2}{|l|}{ Breast cancer liver metastasis } & $\mathrm{n}$ & $\%$ \\
\hline \multicolumn{2}{|l|}{ Mean age (M) } & $59 \pm 11.34$ & (56) \\
\hline \multicolumn{2}{|c|}{ Mean time-interval following primary tumour-years (M) } & 3.9 & $(3.5)$ \\
\hline \multicolumn{4}{|l|}{ Distribution } \\
\hline & Unilateral & 7 & 63.6 \\
\hline & Bilateral & 4 & 36.4 \\
\hline \multicolumn{4}{|l|}{ Number of lesions } \\
\hline & one & 4 & 36.4 \\
\hline & two & 2 & 18.2 \\
\hline & three or more & 5 & 45.5 \\
\hline \multicolumn{2}{|c|}{ Mean maximum tumor size $[\mathrm{mm}](\mathrm{M})$} & 41.3 & 44 \\
\hline \multicolumn{4}{|l|}{ (measured in the biggest diameter) } \\
\hline \multicolumn{2}{|l|}{ Enlarged regional lymph nodes } & 1 & 9.1 \\
\hline \multicolumn{2}{|l|}{ Infiltration of hepatic venous } & 1 & 9.1 \\
\hline \multicolumn{2}{|l|}{ Infiltration of biliary tracts } & 0 & 0 \\
\hline
\end{tabular}

(M) - Median

Table 2. Characteristics of liver resection $(n=11)$

\begin{tabular}{|c|c|c|c|}
\hline & & $\mathrm{n}$ & $\%$ \\
\hline \multicolumn{4}{|l|}{ Extend of resection } \\
\hline & Limited resection ( $<3$ segments) & 8 & 72.7 \\
\hline & Major resection ( $\geq 3$ segments) & 2 & 18.2 \\
\hline \multicolumn{4}{|l|}{ Type of resection } \\
\hline & Left- or Right haemihepatectomy & 2 & 18.2 \\
\hline & Bisegmentectomy & 2 & 18.2 \\
\hline & Non-anatomical/wedge resection & 6 & 54.5 \\
\hline \multicolumn{4}{|l|}{ Thermal ablation } \\
\hline & With liver resection & 4 & 36.4 \\
\hline & alone & 1 & 9.1 \\
\hline
\end{tabular}

Post-operative and long-term outcome. Following surgery, early complications such as haemorrhage, pneumothorax, respiratory and circulation insufficiency, were not observed in the studied group. No re-laparotomies were performed. Late complications - wound infection, biliary leakage or failure of the remaining liver tissue after resection, were not present. The average time of hospitalization was 13.27 days. All patients were discharged in general good condition.

A 5-year overall survival (OS) and disease-free survival was attained by one patient, which equals $9.1 \%$ of all the study group. This patient had a single, $10-\mathrm{mm}$ lesion localized in the right lobe, but the female patient did not required postoperative chemotherapy; however, tamoxifen was administered after mastectomy. 4 (36.4\%) had OS=1 year, DFS - a few months. One patient (9.1\%) died from disseminated disease 6 months after surgery; the patient intraoperatively qualified only for thermal ablation, not liver resection. 5 patients (45.5\%) died before attaining 5 -year OS, but precise data was unavailable to the authors. One patient (9.1\%) died 6 months after, but due to reasons unconnected with breast cancer - a severe brain injury. After surgery, 5 patients (45.5\%) underwent postoperative chemotherapy, and 4 patients hormonal therapy - tamoxifen. None of the patients - pre- or post-operatively required targeted therapy for her-2-NEU receptors.

After surgery, none of patients underwent re-resection for recurrent BCLM metastasis.

\section{DISCUSSION}

Liver resection has proved its efficacy as treatment for isolated liver metastasis of colon cancer (CRLM) and neuroendocrine tumours $[17,18]$. There are many cohort studies giving scientific evidences that in these conditions, metastasectomy significantly prolongs patients' lives $[17,18]$. Contrarily, in researches concerning non-colorectal, nonneuroendocrine, non-sarcoma (NCNNNS) liver metastasis, where the BCLM of often included, the role of hepatic resection is not well-established [19-21]. The NCNNNS group is non-homogenous, where tumours have a different primary prognosis, methods of treatment, and response for chemotherapy; thus, comparing BCLM with liver metastasis from other neoplasms could be difficult and lead to incorrect conclusions [22]. This fact should be taken into consideration while analysing results in the literature.

Although there are inceasingly more studies concerning the surgical treatment of BCLM $[6,8,9,16,20,21,22,23]$, no surgical association has mapped-out guidelines which may optimize the role of BCLM metastasectomy; therefore, the resection of metastatic breast cancer remains controversial [22]. The process of qualification to hepatic resection and course of operation are based on surgeons' experience and intuition. Metastatic breast cancer is a systemic disease and requires multidisciplinary, individual approach [22], which was the inspiration for the presented study.

There are several reasons to consider liver resection for BCLM. The large, multi-centre study by Adam et al. [9] showed that metastasectomy in patients with BCLM has comparable results with hepatic resection performed for colorectal or neuroendocrine liver metastasis [24, 25]. There are also promising results reported in a study comparing survival in patients with BCLM treated by metastasectomy, or by conventional systemic therapy alone [21]. The median overall survival was respectively 50 vs. 45 months; however, the difference was not statistically significant. This might have been caused by patient selection to specific study groups. Despite the above results, the specific group of patients could benefit from liver resection for BCLM - in patients with little hepatic lesions and who underwent $\mathrm{R} 0$ resection the risk of recurrence was the lowest [20]. In patients with triple negative receptors (non-E, non-P, non-HER2-neu receptors), the hepatic resection was likely more beneficial due to drug-resistance of this type of tumour, and insufficient 
systemic therapy [21]. The performance of liver resection is recommended in patients with metachronous BCLM who have had a long disease-free period, in which the metastatic burden is low and liver tumours can be resected with a negative surgical margin $[16,23]$.

Surgical treatment of BCLM in patients' extra-hepatic disease should be performed based on an important difference in survival rates: in isolated bone metastasis the median overall survival is 24-48 months [26], the BCLM presents a median OS of 16.5-26 months [27]. This means that liver resection may prolong a patient's life, even if bone lesions are presented, and especially if patients are receiving additional therapy dedicated to treat bone metastasis. Some authors describe the efficacy of high-dose of tamoxifen administered in breast cancer bone metastasis with positive E-receptors or oral bisphosphonates in improving survival $[28,29,30]$. Women following BCT with current BCLM present higher expectations concerning further treatment which allows for better control and screening following the primary operation [31] which, with regard to detailed longterm follow-up, would be a significant factor in selecting patients with early liver metastasis and expanding the pool of resectable patients. On the other hand, there are studies relating to performing radionuclide therapy with Sm-153; the evidence for increasing survival is limited and the painrelief effect is promising [32]. The above researches establish the role of BCLM-patient selection for surgery, while the presence of bone metastasis is not an absolute exclusion criterion from hepatic resection. Surgery may provide increased survival with an acceptable quality of life in this group of patients.

The mean time of observing recurrence in patients undergoing resection for BCLM was 2.5 years. The type of previously performed mastectomy did not impact on survival after hepatic resection, except axillary lymp-node status at the time of breast surgery, which is in accordance with results presented in the literature $[23,33]$. No repeated hepatectomies were performed in cases of recurrence, a situation associated with advanced extra-hepatic disease and/or impossibility to gain oncologically radical surgery. The above facts should prompt consideration of screening after metastasectomy; there are even studies proving that MRI scans, PET-CT scans and computed tomography are widely useful in detecting breast cancer metastasis [34]; however, none of them touch on BCLM recurrence. This aspect undoubtedly requires more multi-institutional research to optimize the process of qualification to liver resection.

There are several limitations to the presented study: 1) the retrospective character and single-centre research with small sample-size; 2) the selection bias may also have had an influence on the results; 3 ) the role of ethical dilemma surgical procedures in patients with disseminated disease (while metastasis in other organs can not be exclude) may worsen the patient's outcome and shorten the overall survival rate.

To improve patients' outcome, more multi-institutional, randomized studies are needed.

\section{CONCLUSIONS}

There is still much to be improved in the field of treating breast cancer liver metastases patients in Poland. Factors significantly increasing the chance for a successful hepatic resection in patients with breast cancer liver metastases are: small, single lesions resected with R0 margin, longer period between mastectomy and metastasectomy, positive hormonal $\mathrm{E}$ or $\mathrm{P}$ receptors, no existence of extra-hepatic disease and general good condition. The need for post-operative screening and follow-up of breast cancer women in Poland is crucial. Furthermore, the authors urge oncological centres to assess BCLM patients more openly and qualify them for hepatic resection along with adjuvant systemic treatment. Based on this study and literature review, liver resection is recommend as the only treatment significantly prolonging life in this selected group of patients. However, recognizing the limitations of this study, it is acknowledged that the reason for a future prospective randomized controlled trial treatment of BCLM patients by hepatic resection vs. systemic treatment alone. There are particular questions which underlie the importance of this topic, and thus with new drugs being introduced, would they prove an aid to surgical therapy, or can the OSR be achieved in BCLM as in CRLM patients?

\section{REFERENCES}

1. Polish National Registry of Cancer, http://onkologia.org.pl/nowotworypiersi-kobiet/

2. Banas T, Juszczyk G, Pitynski K, Nieweglowska D, Ludwin A, Czerw A. Incidence and mortality rates in breast, corpus uteri, and ovarian cancers in Poland (1980-2013): an analysis of population-based data in relation to socioeconomic changes. OnkoTargets Therapy 2016; 7 Sept.

3. Carsoso F, Costa A, Norton L, Cameron D, Cuffer T, Fallowfield L, et al. $1^{\text {st }}$ International consensus guidelines for advanced breast cancer (ABC1). Breast 2012; 21: 242-252.

4. Jemal A, Siegel R, Ward E, Murrau T, Xu J, Thun MJ. Cancer Statistics, 2007. CA Cancer J Clin. 2007; 57: 43-66.

5. Zielinski J, Jaworski R, Irga-Jaworska N, Haponiuk I, Jaskiewicz J. The significance of ductoscopy of mammary ducts in the diagnostics of breast neoplasms. Videosurgery Miniinv 2015; 10 (1): 79-86.

6. Vertriest C, Berardi G, Tomassini F, Broucke RV, Depypere H, Cocquyt $\mathrm{V}$, et al. Resection of Single Metachronous Liver Metastases from Breast Cancer Stage I-II Yeld Excellent Overall and Disease-Free Survival. Single Centre Experience and Review of the Literature. Dig Surg. 2015; 32: 52-59.

7. Lee YT. Breast carcinoma: pattern of recurrence and metastasis after mastectomy. Am J Clin Oncol. 1984; 7: 443-9.

8. Ruiz A, Wicherts DA, Sebagh M, Giacchetti S, Castro-Benitez C, van Hillegersberg R, et al. Predictive Profile-Normogram for Liver Resection for Breast Cancer Metastases: An Aggressive Approach with Promising Results Ann Surg Oncol. 2016 Aug 29.

9. Adam R, Chiche L, Aloia, Ellias D, Salmon RJ, et al. Hepatic Resection for Noncolorectal, Nonendocrine Liver Metastases. Analysis of 1452 patients and development of a prognostic model. Ann Surg. 2006; 244: 524-535.

10. Insa A, LLuch A, Prosper F, Marguan I, Marginez-Agullo A, GarciaConde J. Prognostic factors predicting survival from first recurrence in patients with metastatic breast cancer: analysis of 439 patients. Breast Cancer Res Treat. 1999; 56: 67-78.

11. Hoe AL, Royle GT, Taylor I. Breast liver metastases - incidence, diagnosis and outcome. J R Soc Med. 1991; 84: 714-716.

12. Polistina F, Constantin G, Febbraro A, Robusto E, Ambrosino G. Aggressive treatment for hepatic metastases from breast cancer: results from a single center. World J Surg. 2013; 37: 1322-1332.

13. Gonzalez-Angulo AM, Morales-Vasquez F, Hortobagyi GN. Overview of resistance to systemic therapy in patients with breast cancer. Adv Exp Med Biol. 2007; 608: 1-22.

14. Coley HM. Mechanisms and strategies to overcome chemotherapy resistance in metastatic breast cancer. Cancer Treat Rev. 2008; 34: 378-390.

15. Li Z, Kang Y. Emerging therapeutic targets in metastatic progression a focus on breast cancer. Pharmacol Ther. 2016; 161: 79-96. 
16. Margonis G A, Buettner S, Sasaki K, Kim Y, Ratii F, Russolilo N, et al. The role of liver-directed surgery in patients with hepatic metastasis from breast cancer: a multi-institutional analysis. HPB (Oxford) 2016; 18: $700-705$

17. Fong Y, Fortner J, Sun RL, Brennan MF, Blumgart LH. Clinical score for predicting recurrence after hepatic resection for metastatic colorectal cancer Analysis of 1001 consecutive cases. Ann Surg. 1999; 230: 309-318

18. Gurusamy KS, Ramamoorthy R, Sharma D, Davidson BR. Liver resection versus other treatments for neuroendocrine tumors in patients with resectable liver metastases. Cochrane Database Syst Rev. 2009, April 15.

19. Martel G, Hawel J, Rekman J, Croome KP, Bertens K, Balaa FK, Hernandez-Alejandro R. Liver Resection for Non-Colorectal, NonCarcinoid, Non-Sarcoma Metastases: A Multicenter Study; Plos One 2015; March 26.

20. Sabol M, Donat R, Chvalny P, Dyttert D, Palaj J, Durdik S. Surgical management of breast cancer metastases Neoplasma 2014; 61: 5.

21. Sadot E, Ser Yee Lee, Sofocleous CT, Solomon SB, Gonen M, Kingham TP, et al. Hepatic Resection or Ablation for Isolated Breast Cancer Liver Metastasis A case-control Study with Comparison to Medically Treated Patients. Ann Surg. 2016; 264: 147-154.

22. Ruiz A, Castro-Betnitez C, Sebagh M, Giaccetti S, Castro-Santa E, Witchers DA, et al. Repeat Hepatectomy for Breast Cancer Liver Metastases. Ann Surg Oncol. 2015; 22: S1057-S1066.

23. Bacalbasa N, Dima SO, Purtan-Purnichescu R, Herlea V, Popescu I. Role of Surgical Treatment in Breast Cancer Liver Metastases: A Single Center Experience. Anticancer Res. 2014; 34(10): 5563-5568.

24. Frankel TL, D’Angelica MI. Hepatic resection for colorectal metastases Surg Oncol. 2014; 109: 2-9.

25. Lee SY, Cheow PC, Teo JY, et al. Surgical treatment of neuroendocrine liver metastases Int J Hepatol. 2012; 2012: 146590.
26. Saarto T, Janes R, Tenhunen M, Kouri M. Palliative radiotherapy for bone metastases - single-dose radiotherapy is effective. Duodecim 2002; 118: 1889-1894.

27. Pagani O, Senkus E, Wood W, Colleoni M, Cufer T, Kyriakides S, et al. International Guidelines for management of metastatic breast cancer: can metastatic breast cancer be cured? J Natl Cancer Inst. 2010; 102: 456-463.

28. Gabriel I, Olejek A. The value of hysteroscopic-based decision-making in uterine abnormalities during tamoxifen intake in breast cancer patients - preliminary report. Videosurgery Miniinv 2014; 9 (2): $258-261$.

29. Stathopoulos GP, Trafalis D, Kaparelou M. Bone metastasis in breast cancer is treated by high-dose tamoxifen. J BUON 2016 Aug; 21(4):10131015.

30. Rennert G, Pinchev M, Gronich N, Saliba W, Flugelman A, Lavi I, et al. Oral bisphosphonates and improved survival of breast cancer. Clin Cancer Res. 2016 Sept 28.

31. Kamińska M, Ciszewski T, Kukiełka-Budny B. Life quality of women with breast cancer after mastectomy or breast conserving therapy treated with adjuvant chemotherapy. Ann Agric Environ Med. 2015; 22 (4): 724-730.

32. Correa-González L, Arteaga de Murphy C, Pichardo-Romero P, Pedraza-López M, Moreno-García C. (153)Sm-EDTMP for pain relief of bone metastases from prostate and breast cancer and other malignancies. Arch Med Res. 2014; May 45(4): 301-8.

33. Nowikiewicz T, Nowak A, Wiśniewska M, Wiśniewski M, Zegarski W. Diagnostic value of preoperative axillary lymph node ultrasound assessment in patients with breast cancer qualified for sentinel lymph node biopsy. Videosurgery Miniinv. 2015; 10 (2): 170-177.

34. Lei L, Wang X, Chen Z. PET/CT Imaging for Monitoring Recurrence and Evaluating Response to Treatment in Breast Cancer. Adv Clin Exp Med. 2016; 25(2): 377-382. 